\title{
Enter 2D quantum materials
}

\author{
The discovery of long-range magnetic order, strong correlations and superconductivity in 2D materials provides \\ new opportunities to study and control these phenomena.
}

$(2)$

uantum effects can manifest on different length scales. For example, the Stern-Garlach experiment considered a beam of un-polarized magnetic-moment-carrying silver atoms traversing an inhomogeneous magnetic field ${ }^{1}$. Classically one expected a continuous distribution of magnetic-field-deflected atoms to appear in the detector but what one observes is only two different states as a result of angular momentum quantization. This experiment demonstrated the importance of quantum effects at the atomic length scale. At larger length scales many quantum behaviours in solids have been observed over the years, including, soon after the isolation of $2 \mathrm{D}$ materials, the quantum Hall effect in graphene $e^{2,3}$. More recently 2D materials have expanded their quantum reach even further with the demonstration of long-range magnetic order in monolayer materials such as $\mathrm{CrI}_{3}\left(\right.$ refs. $\left.{ }^{4,5}\right)$ and the finding of strong electronic correlations and superconductivity in materials such as twisted bilayer graphene (TBG) ${ }^{6,7}$.

Scientists have been working on magnetism and superconductivity (and their interplay) for a long time and have most commonly used chemical doping, temperature, magnetic field, high pressure and electric current to tune their properties. Van der Waals materials offer a new route: monolayers can be stacked sequentially and the relative twist angle between these layers controlled. The resulting moiré pattern, with its large period, can radically affect electronic properties, as emphasized in a Review from Eva Andrei and Allan MacDonald in this issue of Nature Materials. Finer twist angle control utilizing a tear-and-stack fabrication method enabled transport measurements that revealed correlation-driven insulating states, flat bands and low-temperature superconductivity in $\mathrm{TBG}^{6,7}$. These results stimulated much initial excitement due to a similar phenomenology with known high-temperature superconductors (HTS) as well as the ability to continuously tune the carrier density by electrostatic gating. Andrei and MacDonald compare and

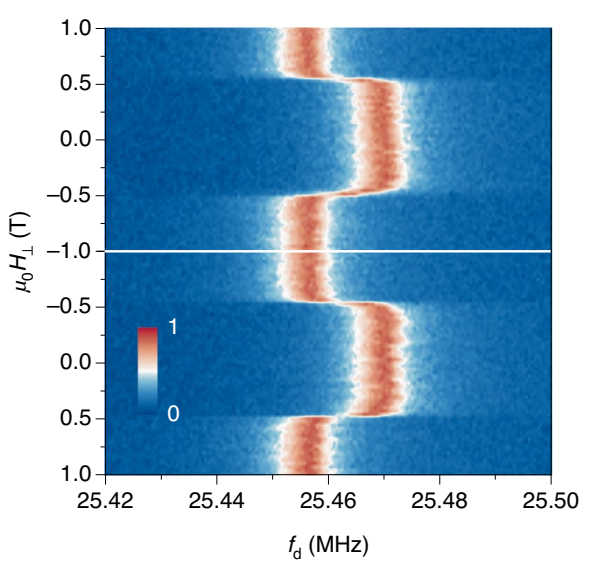

Normalized vibration amplitude of bilayer $\mathrm{Crl}_{3}$ resonator versus driving frequency under an out-of-plane magnetic field $\left(\mu_{0} H_{\perp}\right)$. Credit:

Springer Nature Ltd.

contrast TBG and HTS and expand further on the abundance of interesting physics in TBG - for example, orbital magnetism in which the orbital component of the magnetic moment dominates over the spin component. They also emphasize that further theoretical understanding will rely on achieving more experimental progress. Such progress is currently hampered by unreliable twist angle control but advances are being made and the future appears promising for moiré superlattices.

Magnetism is a subject of perennial fundamental interest with important practical applications, for example in magnetic hard drives. While several platforms exist for studying 2D magnetism, for example thin films and quasi-2D crystals, it was only a few years ago that long-range magnetic order was discovered in $2 \mathrm{D}$ materials ${ }^{4,5}$. This platform presents unique routes for the control of magnetic order through electrical gating, stacking and heterostructuring, as emphasized by Xiaodong $\mathrm{Xu}$ and collaborators in their Review in this issue. The authors discuss different 2D materials exhibiting diverse types of magnetic order and the experimental probes used to detect such order. At the same time, they outline the pressing need for new air-stable, room-temperature $2 \mathrm{D}$ magnets and probes to directly characterize them in the monolayer limit. The potential for realizing new states through the interplay of magnetism and topology in 2D materials is also highlighted, for example skyrmions in moiré superlattices.

We also include in this Focus issue two original research letters on 2D magnets. To address critical magnetic fluctuations that dominate around the ordering temperatures of magnets, Jie Shan and colleagues report in a Letter further development of a magneto-optical technique to achieve sensitivity to the magnetization state of a 2D material on short time and length scales. Practically, this technical advance enabled switching of magnetic states by electrostatic-gating-controlled critical fluctuations alone. A Letter by Kin-Fai Mak and colleagues reports on the detection of magnetic states by mechanical resonances (pictured), with potential practical implications for magneto-strain sensors. Both of these works on 2D magnets underline the connection between fundamental, technical and practical advances in the field of 2D magnetism.

We have not by any means covered all aspects of $2 \mathrm{D}$ quantum materials here for example, much work is being done on interlayer excitons ${ }^{8}$ and strong correlations in other twisted systems. Nevertheless, we hope this Focus issue will whet the appetite of scientists planning their next experiment.

Published online: 18 November 2020 https://doi.org/10.1038/s41563-020-00867-3

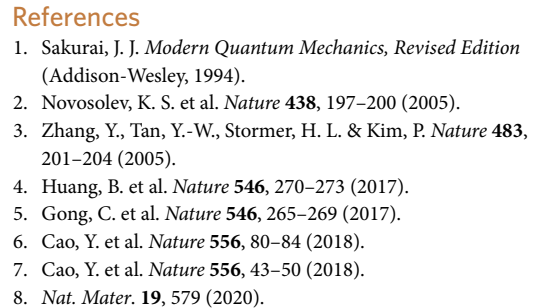

